\title{
Comparison of the Efficacy of Intravenous, Subcutaneous, and Suppository Morphine in Reducing Post Hysterectomy Pain
}

\author{
Sheida Shabanian' $^{(\mathbb{1}}$, Ali Ahmadii $^{\circledR}$, Razieh Mohammadi ${ }^{\circledR}{ }^{\circledR}$, Gholamreza Shabanian $^{*}{ }^{(\mathbb{D}}$ \\ 'Department of Obstetrics and Gynecology, Shahrekord University of Medical Sciences, Shahrekord, Iran \\ ${ }^{2}$ Department of Statistics, Shahrekord University of Medical Sciences, Shahrekord, Iran \\ ${ }^{3}$ Shahrekord University of Medical Sciences, Shahrekord, Iran \\ ${ }^{4}$ Department of Anesthesia, Shahrekord University of Medical Sciences, Shahrekord, Iran
}

*Corresponding Author: Gholamreza Shabanian, Department of Anesthesia, Ayatollah Kashani Hospital, Shahrekord University of Medical Sciences, Iran, Tel: +989133827110, Email: gshabanian@yahoo.com

\begin{abstract}
Background and aims: Postoperative pain has always been considered by surgeons because of its various complications. The aim of this study was to compare the effect of intravenous, subcutaneous and suppository morphine in reducing post-hysterectomy pain.

Materials and Methods: In this clinical trial, 90 patients undergoing hysterectomy were randomized into three groups of 30 each using simple randomization, namely, intravenous, subcutaneous, and suppository morphine (10 mg). Before intervention and 4, 8, 12, and 16 hours after intervention, pain intensity was measured using visual analogue scale (VAS). Relative frequency of nausea, vomiting, itching, bradypnea, and apnea in all groups was recorded. Data were analyzed by SPSS version16.0.

Results: Mean pain severity at 0 hour postoperatively $(P=0.004)$, 4 hours postoperatively $(P=0.009), 8$ hours postoperatively $(P=0.009)$, and 12 hours postoperatively $(P=0.001)$ was significantly higher in the suppository morphine group than in the other two groups. There was no significant difference in pain severity at 16 hours postoperatively among the three groups $(P=0.446)$. According to the results of repeated measures ANOVA, changes in pain severity at the five intervals were statistically significant in all three groups (subcutaneous, intravenous, and suppository morphine groups) $(P<0.001)$. There was also a statistically significant difference in pain severity at the studied intervals among the three groups $(P<0.001)$. The frequency of nausea $(P=0.05)$ and vomiting $(P=0.84)$ was higher in the suppository group than in the other two groups, although the difference was not statistically significant $(P=0.05)$.

Conclusion: The results of this study indicated better efficacy of subcutaneous and intravenous morphine in reducing post-hysterectomy pain compared with suppository morphine.

Keywords: Morphine, Hysterectomy, Pain
\end{abstract}

Received: 11 June 2019, Accepted: 16 September 2019, ePublished: 29 December 2020

\section{Introduction}

Pain is considered as one of the vital signs that if neglected leads to many negative consequences (1). Pain is an important determinant of health-related quality of life that causes postoperative cardiovascular, gastrointestinal, psychological and pulmonary complications and disability and increases hospitalization and pain medication use (2-8). Treatment of acute pain and postoperative pain is important in health care settings. In recent years, many advances have been made in understanding the process of pain development and the development of pain relievers and pain relief techniques (9). Oral and intravenous administrations are methods of prescribing analgesic drugs for the control of postoperative acute pain. Oral administration of analgesic drugs is not suitable for controlling moderate to severe postoperative acute pain because it does not provide rapid and efficient pain relief $(10,11)$.

Hysterectomy is a common gynecological surgery that causes moderate to severe postoperative pain (12). Hence, the optimal management of post-hysterectomy pain is essential for preventing the development of chronic pain. Various treatments and analgesic administration routes can lead to various outcomes such as changes in visual analogue scale (VAS) scores, sedation, and even side effects $(11,13)$. A study that compared the type of opioids used showed that oral administration was associated with delayed onset of analgesia and reduced patient satisfaction but needed shorter time to be accomplished. In contrast, intravenous morphine titration caused rapid and adequate pain relief (11). Considering that the opioid receptors are located at peripheral nerve terminals (14), the peripheral application of opioids is of clinical importance. In addition, the efficacy of intravenous, subcutaneous, and

(C) 2020 The Author(s); Published by Shahrekord University of Medical Sciences. This is an open-access article distributed under the terms of the Creative Commons Attribution License (http://creativecommons.org/licenses/by/4.0), which permits unrestricted use, distribution, and reproduction in any medium, provided the original work is properly cited. 
suppository morphine in reducing postoperative pain has not been investigated. Therefore, we decided to compare subcutaneous, intravenous, and suppository administrations of morphine to compare their efficacy and complications.

\section{Materials and Methods}

In this triple-blind clinical trial, patients referred for hysterectomy to Hajar and Kashani Hospitals in Shahrekord in 2018 were enrolled.

In this clinical trial, 90 patients undergoing hysterectomy were randomized into three groups of 30 each using simple randomization, namely, intravenous, subcutaneous, and suppository morphine $(10 \mathrm{mg})$. To this end, we used a simple randomized list with numerical sequential unique identifiers.

The patients undergoing hysterectomy with a body weight of 50 to $80 \mathrm{~kg}$ and a willingness to participate in the study were included. The exclusion criteria were addiction, allergies to morphine and similar compounds, hepatic and renal failure, pulmonary dysfunction, use of monoamine oxidase inhibitors (MAOIs), and a history of anesthesia for any reason.

The sample size was calculated to be 28 for each group according to a similar study (15) using STATA software, considering the type 1 error $=0.05$, Power $=0.8$, Delta $=1.30$, standard deviation=1, $M_{1}=5.8$, and $M_{2}=4.5$. Patients were selected by simple sampling method and then were randomly allocated to three groups using Random Allocation software.

Blinding is a measure in randomized controlled trials to reduce detection and performance bias. In the current study, patients, interventional physicians, and evaluators did not know the status of the groups.

Then, the patients were randomly assigned to three groups (A, B, and C groups) of 30 each. The patients were given adequate explanations regarding the VAS before entering the operating room. After entering the operating room, patients were monitored for blood pressure, heart rate, and pulse oximetry. Patients received fentanyl $(3 \mu \mathrm{g} /$ $\mathrm{kg})$, thiopental sodium $(5 \mathrm{mg} / \mathrm{kg})$, and atracurium $(0.5 \mathrm{mg} /$ $\mathrm{kg}$ ) and underwent general anesthesia. Then, the patients were intubated and underwent surgery. After surgery and transfer to the recovery room, one of the administration methods of morphine was used to control pain in each group. Group A received intravenous morphine (10 mg), group $B$ received subcutaneous morphine $(10 \mathrm{mg})$, and group C received suppository morphine (10 mg) (16). Morphine was given by the nurse at 0 hour (immediately after transfer to recovery). At 4, 8, 12, and 16 hours after surgery, morphine was re-administered if the patient had pain. Pain intensity was assessed at $0,4,8,12$, and 16 hours after surgery using VAS. For patients whose pain was not relieved, pethidine was used and pain intensity was recorded in the checklist. If complications occurred, the necessary measures would be taken. Patients' information including pain, nausea, vomiting, itching, bradypnea, and apnea was collected by checklist. Data were analyzed by SPSS version 16.0 using descriptive statistics (frequency, percentage, mean, and SD), repeated measures ANOVA, ANOVA, and chi-squared test. In repeated measures ANOVA, we included groups, time intervals, and interaction between them and reported interaction as an important subject. $P<0.05$ was considered the significance level.

\section{Results}

In this study, there was no difference among groups in terms of demographic variables. The flowchart of the randomized clinical trial is shown in Figure 1.

Mean and standard deviation of VAS at different intervals after surgery in each group are reported in Table 1 and illustrated in Figure 2. The lowest pain severity at $0,4,8,12$, and 16 hours was observed in the intravenous morphine group (Table 1).

Based on the findings (Table 1), mean pain severity at 0 hour postoperatively $(P=0.004), 4$ hours postoperatively $(P=0.009), 8$ hours postoperatively $(P=0.009)$ and 12 hours postoperatively $(P=0.001)$ was significantly different among the three groups (suppository morphine, subcutaneous morphine, and intravenous morphine), indicating that the mean pain severity at $0,4,8$ and 12 hours postoperatively was significantly higher in the suppository morphine group than in the other two groups. There was no significant difference in pain severity at 16 hours postoperatively among the three groups $(P=0.446)$.

Table 1. The intensity of pain at different intervals in the studied groups

\begin{tabular}{|c|c|c|c|c|}
\hline \multirow{2}{*}{$\begin{array}{l}\text { Interval } \\
\text { (h) }\end{array}$} & \multicolumn{3}{|c|}{ Mean $\pm S$ D } & \multirow{2}{*}{$P$ value } \\
\hline & Suppository morphine & Intravenous morphine & Subcutaneous morphine & \\
\hline 0 & $9.06 \pm 1.08$ & $7.43 \pm 2.14$ & $7.50 \pm 2.75$ & 0.004 \\
\hline 4 & $7.10 \pm 1.88$ & $5.36 \pm 1.88$ & $6.56 \pm 2.69$ & 0.009 \\
\hline 8 & $6.30 \pm 1.53$ & $4.53 \pm 1.88$ & $5.80 \pm 2.07$ & 0.001 \\
\hline 12 & $4.36 \pm 1.58$ & $3.23 \pm 1.63$ & $2.83 \pm 1.51$ & 0.001 \\
\hline 16 & $1.70 \pm 1.11$ & $1.33 \pm 1.12$ & $1.50 \pm 1.12$ & 0.446 \\
\hline$P$ vvalue $^{\mathrm{a}}$ & $<0.001$ & $<0.001$ & $<0.001$ & \\
\hline
\end{tabular}

a Repeated measures ANOVA. 


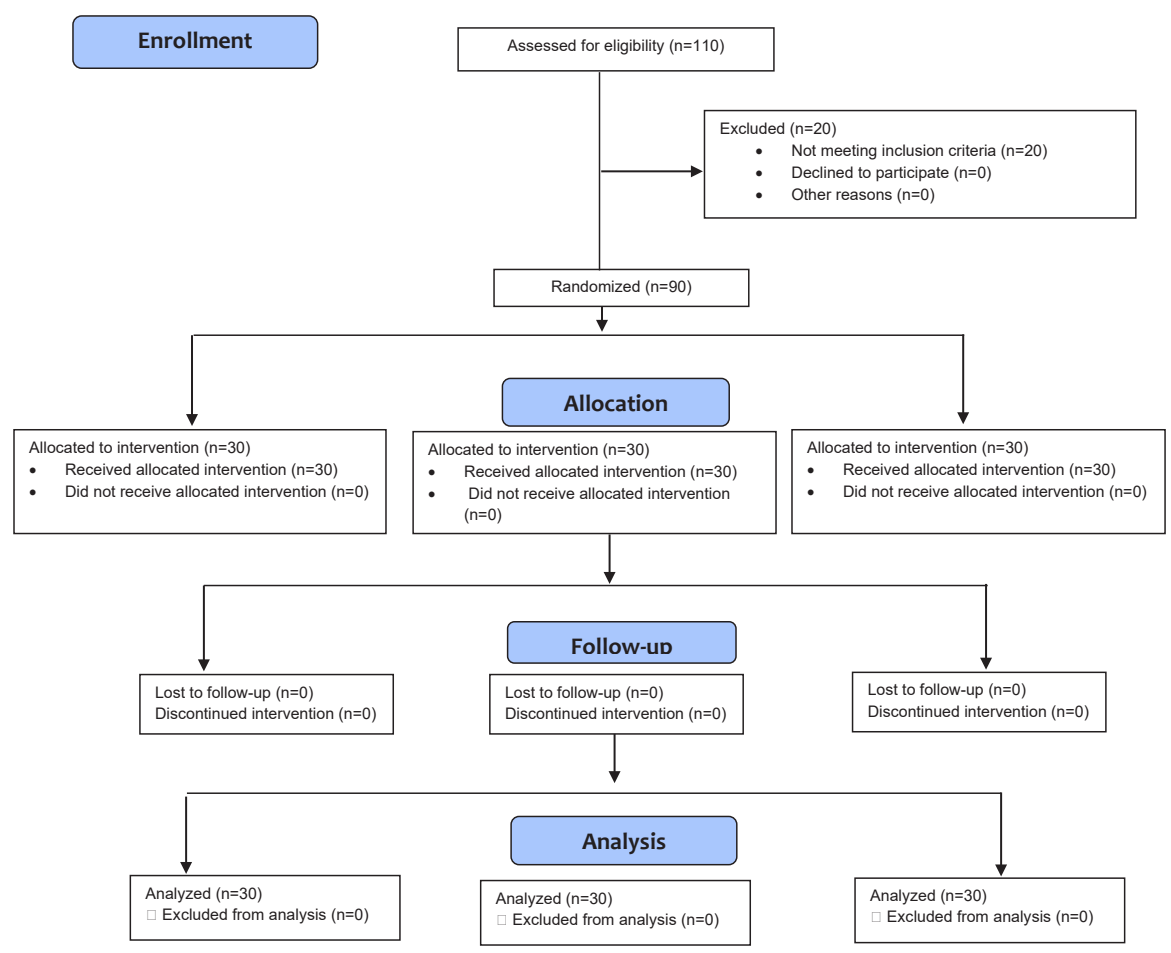

Figure 1. Flowchart of participant enrollment.

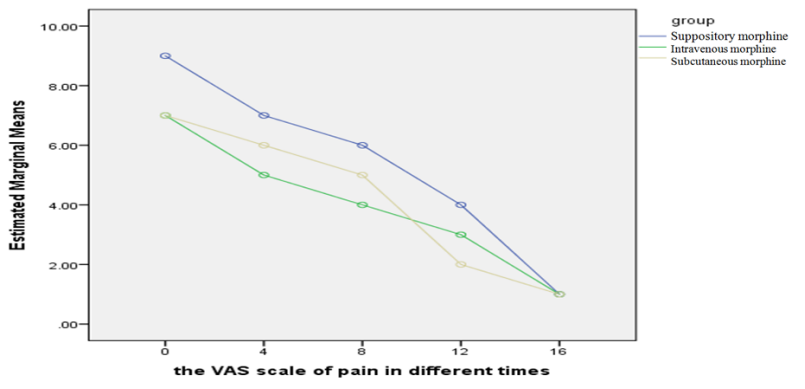

Figure 2. Pain intensity at different intervals in the studied groups based on the visual analogue scale.

According to the results of repeated measures ANOVA, there was a statistically significant difference in pain severity at the five intervals $(0,4,8,12$, and, 16 hours postoperatively) among the three groups (suppository morphine, subcutaneous morphine, and intravenous morphine) $(P<0.001)$ (Table 1$)$. The results of this test also showed that there was a significant difference in pain severity changes among the three groups (suppository morphine, subcutaneous morphine, and intravenous morphine) $(P<0.001)$, indicating that the changes were significantly higher in the suppository morphine group than in the intravenous morphine group $(P<0.001)$ and the subcutaneous morphine group $(P=0.009)$ (Table 2$)$.

The frequency of nausea and vomiting was higher in the morphine suppository group than in the intravenous morphine and subcutaneous morphine groups but there was no significant difference between the studied groups $(P>0.05)$. None of the patients had itching, apnea, or tachycardia.
The average dose of morphine administered in the morphine suppository group (3.3) was higher than that in the subcutaneous (2.9) and intravenous morphine (2.7) groups.

Ten patients in the suppository morphine group, 8 patients in the subcutaneous morphine, and 2 patients in the intravenous morphine group received a single dose of pethidine (Table 3).

\section{Discussion}

The aim of this study was to compare the efficiency of intravenous, subcutaneous, and suppository morphine in reducing post-hysterectomy pain. The mean postoperative pain score was significantly lower in the subcutaneous and intravenous morphine groups than in the suppository morphine group; however, the mean postoperative pain score in subcutaneous and intravenous morphine groups was not significantly different. In addition, the average number of doses of morphine was higher in the morphine suppositorygroup than in the intravenous and subcutaneous morphine groups. Ten patients in the suppository morphine group, 8 patients in the subcutaneous morphine group, and 2 patients in the intravenous morphine group received pethidine. The review of literature shows that no study has yet been conducted to compare the efficacy of the three administration methods. However, we discussed similar studies in this section. In line with the results of this study, a study reported that postoperative pain was lower in the suppository acetaminophen group than in the intravenous acetaminophen group in children and request for additional analgesic medication was less in the 
Table 2. Comparison of postoperative pain intensity among the studied groups

\begin{tabular}{|c|c|c|c|c|}
\hline \multicolumn{2}{|l|}{ Groups } & \multirow{2}{*}{ Mean difference (I-J) } & \multirow{2}{*}{ Standard error } & \multirow{2}{*}{$P$ value } \\
\hline (I) Group & (J) Group & & & \\
\hline \multirow{2}{*}{ Suppository morphine } & Intravenous morphine & 1.32 & 0.286 & $<0.001^{\mathrm{a}}$ \\
\hline & Subcutaneous morphine & 0.87 & 0.286 & $0.009^{b}$ \\
\hline \multirow{2}{*}{ Intravenous morphine } & Suppository morphine & -1.32 & 0.286 & $<0.001^{\mathrm{a}}$ \\
\hline & Subcutaneous morphine & -0.45 & 0.286 & 0.351 \\
\hline \multirow{2}{*}{ Subcutaneous morphine } & Suppository morphine & -0.87 & 0.286 & $0.009^{b}$ \\
\hline & Intravenous morphine & 0.45 & 0.286 & 0.351 \\
\hline
\end{tabular}

${ }^{\text {a } P<0.001 \text { and }^{\text {b }} P<0.01}$

Table 3. Comparison of the frequency of nausea and vomiting and single-dose administration of pethidine among the studied groups

\begin{tabular}{|c|c|c|c|c|c|}
\hline Variables & & $\begin{array}{l}\text { Suppository morphine } \\
\text { (number) }\end{array}$ & $\begin{array}{l}\text { Intravenous morphine } \\
\text { (number) }\end{array}$ & $\begin{array}{l}\text { Subcutaneous morphine } \\
\text { (number) }\end{array}$ & $P$ value $^{a}$ \\
\hline \multirow{5}{*}{ Vomiting } & Never & 17 & 21 & 18 & \multirow{5}{*}{0.057} \\
\hline & Once & 3 & 3 & 8 & \\
\hline & Twice & 6 & 2 & 2 & \\
\hline & Three times & 4 & 4 & 0 & \\
\hline & Five times & 0 & 0 & 2 & \\
\hline \multirow{3}{*}{ Nausea } & Never & 15 & 15 & 14 & \multirow{3}{*}{0.84} \\
\hline & Once & 15 & 13 & 2 & \\
\hline & Twice & 14 & 14 & 2 & \\
\hline \multicolumn{2}{|c|}{$\begin{array}{l}\text { Single-dose administration } \\
\text { of pethidine }\end{array}$} & 10 & 2 & 8 & - \\
\hline
\end{tabular}

suppository group than in the intravenous group (17).

Khalili et al compared the effects of intravenous morphine and suppository morphine. Based on the results, the mean pain intensity score at 22 hours after surgery was not significantly different between the two groups. Therefore, injected and suppository morphine acted similarly in reducing postoperative pain. In addition, there was no significant difference in the level of drowsiness at any of the studied intervals between the two groups (18). Injected morphine was administered by patient controlled analgesia (PCA) and morphine was administered at different concentrations. The differences between the results of this study and those obtained by Khalil et al are due to the administration route and frequency of administration of morphine.

Giordano et al conducted a study on the comparison of postoperative analgesic effects of intravenous, intramuscular, subcutaneous, and oral transmucosal buprenorphine in cats. Their results showed that intravenous and intramuscular prescription of buprenorphine provided better postoperative pain relief than subcutaneous and oral transmucosal administration of the drug (19). Another study reported that continuous epidural morphine did not produce the expected effect in the patients undergoing posterior lumbar interbody fusion and continuous subcutaneous morphine led to some analgesic effects with fewer complications (20). In a study conducted by Barnhart et al, intravenous morphine at $0.5 \mathrm{mg} / \mathrm{kg}$ and suppository morphine at $5 \mathrm{mg} / \mathrm{kg}$ produced similar analgesic effects and Than intravenous morphine (21). However, morphine dose was lower in the suppository morphine group in the present study than in a study conducted by Barnhart et al (21), to which the lower efficacy of suppository morphine in our study could be attributed.

Overall, the analgesic effects of opioids occur through the activation of opioid receptors in the central nervous system. Opioid agonists produce analgesia by binding to specific receptors coupled to $G$ protein and cause cellular hyperpolarization. The majority of clinically relevant opioid analgesics bind to MOP receptors in both the central and peripheral nervous system in an agonist manner to elicit analgesia (22). However, in the present study, the administration of suppository morphine had less analgesic effects than intravenous and subcutaneous morphine, but its administration can be varied depending on patient's preference and pain intensity.

\section{Conclusion}

The mean postoperative pain score was lower in the subcutaneous and intravenous morphine groups than in the morphine suppository group. In addition, the frequency of nausea and vomiting was higher in the morphine suppository group than in the other groups. Considering 
the convenience of subcutaneous administration of morphine and its fewer complications, this method is suggested to relieve post-hysterectomy pain.

Conflict of Interests

The authors declare that there is no conflict of interests.

Ethical Approval

The protocol of the clinical trial was approved by the Ethics Committee of Shahrekord University of Medical Sciences (IR. SKUMS.REC.1394.221) and written consent to participate in the study was obtained from patients.

Authors Contribution

Conception and design, acquisition of data, analysis and interpretation of data done by SS and GS. Drafting of the manuscript performed by RM and GS. Critical revision of the manuscript for important intellectual content and statistical analysis done by $A A$. $A B$ and $A D$ contributed in acquisition of data. The final draft approved by all authors.

\section{Funding/Support}

None.

\section{Acknowledgments}

This article was derived from a research project funded by the Research and Technology Deputy of Shahrekord University of Medical Sciences (Approval Number: 2033).

\section{References}

1. Morone NE, Weiner DK. Pain as the fifth vital sign: exposing the vital need for pain education. Clin Ther. 2013;35(11):172832. doi: 10.1016/j.clinthera.2013.10.001.

2. Mullady DK, Yadav D, Amann ST, O'Connell MR, Barmada MM, Elta $\mathrm{GH}$, et al. Type of pain, pain-associated complications, quality of life, disability and resource utilisation in chronic pancreatitis: a prospective cohort study. Gut. 2011;60(1):7784. doi: 10.1136/gut.2010.213835.

3. Zubrzycki M, Liebold A, Skrabal C, Reinelt H, Ziegler M, Perdas E, et al. Assessment and pathophysiology of pain in cardiac surgery. J Pain Res. 2018;11:1599-611. doi: 10.2147/ jpr.s162067.

4. Fayaz A, Ayis S, Panesar SS, Langford RM, Donaldson LJ. Assessing the relationship between chronic pain and cardiovascular disease: a systematic review and metaanalysis. Scand J Pain. 2016;13:76-90. doi: 10.1016/j. sjpain.2016.06.005.

5. Kolacz J, Porges SW. Chronic diffuse pain and functional gastrointestinal disorders after traumatic stress: pathophysiology through a polyvagal perspective. Front Med (Lausanne). 2018;5:145. doi: 10.3389/fmed.2018.00145.

6. Shea RA, Brooks JA, Dayhoff NE, Keck J. Pain intensity and postoperative pulmonary complications among the elderly after abdominal surgery. Heart Lung. 2002;31(6):440-9. doi: $10.1067 / \mathrm{mhl} .2002 .129449$.

7. Gorczyca R, Filip R, Walczak E. Psychological aspects of pain. Ann Agric Environ Med. 2013;Spec no. 1:23-7.

8. Vadivelu N, Kai AM, Kodumudi G, Babayan K, Fontes M, Burg
MM. Pain and psychology-a reciprocal relationship. Ochsner J. 2017;17(2):173-80.

9. Pogatzki-Zahn EM, Segelcke D, Schug SA. Postoperative painfrom mechanisms to treatment. Pain Rep. 2017;2(2):e588. doi: 10.1097/pr9.0000000000000588.

10. Becker DE. Pain management: Part 1: managing acute and postoperative dental pain. Anesth Prog. 2010;57(2):67-78. doi: 10.2344/0003-3006-57.2.67.

11. Pan Z, Qi Y, Wen Y, Chen L. Intravenous morphine titration vs. oral hydrocodone/acetaminophen for adults with lower extremity displaced fracture in an emergency department setting: a randomized controlled trial. Exp Ther Med. 2018;16(4):3674-9. doi: 10.3892/etm.2018.6606.

12. Brandsborg B. Pain following hysterectomy: epidemiological and clinical aspects. Dan Med J. 2012;59(1):B4374.

13. Raghvendra KP, Thapa D, Mitra S, Ahuja V, Gombar S, Huria A. Postoperative pain relief following hysterectomy: a randomized controlled trial. J Midlife Health. 2016;7(2):65-8. doi: 10.4103/0976-7800.185327.

14. Stein C. Opioid receptors on peripheral sensory neurons. In: Madame Curie Bioscience Database. Austin, TX: Landes Bioscience; 2000-2013.

15. Soltani H, Hashemi S, Izadi A, Dereimi KZ, Safaei $M$, Zarinjouei $\mathrm{H}$. effect Of pre-incisional subcutaneous infiltration of morphine on pain intensity after upper abdominal surgery: pb213. Pain Practice. 2009;9:152.

16. Katzung BG, Masters SB, Trevor AJ. Basic \& Clinical Pharmacology. USA: McGraw-Hill Medical; 2016.

17. Haddadi S, Marzban S, Karami MS, Heidarzadeh A, Parvizi A, Naderi Nabi B. Comparing the duration of the analgesic effects of intravenous and rectal acetaminophen following tonsillectomy in children. Anesth Pain Med. 2014;4(1):e13175. doi: 10.5812/aapm.13175.

18. Khalili GR, Heidari S, Sorani Heidari M. Comparison of intravenous morphine with morphine sulfate suppository in control of pain after knee and hip arthroplasty. J Babol Univ Med Sci. 2013;15(6):19-24. doi: 10.18869/acadpub. jbums.15.6.19. [Persian].

19. Giordano T, Steagall PV, Ferreira TH, Minto BW, de Sá Lorena SE, Brondani J, et al. Postoperative analgesic effects of intravenous, intramuscular, subcutaneous or oral transmucosal buprenorphine administered to cats undergoing ovariohysterectomy. Vet Anaesth Analg. 2010;37(4):357-66. doi: 10.1111/j.1467-2995.2010.00541.x.

20. Yukawa $Y$, Kato F, Ito K, Terashima T, Horie Y. A prospective randomized study of preemptive analgesia for postoperative pain in the patients undergoing posterior lumbar interbody fusion: continuous subcutaneous morphine, continuous epidural morphine, and diclofenac sodium. Spine (Phila Pa 1976). 2005;30(21):2357-61. doi: 10.1097/01. brs.0000184377.31427.fa.

21. Barnhart MD, Hubbell JA, Muir WW, Sams RA, Bednarski RM. Pharmacokinetics, pharmacodynamics, and analgesic effects of morphine after rectal, intramuscular, and intravenous administration in dogs. Am J Vet Res. 2000;61(1):24-8. doi: 10.2460/ajvr.2000.61.24.

22. Pathan H, Williams J. Basic opioid pharmacology: an update. Br J Pain. 2012;6(1):11-6. doi: 10.1177/2049463712438493. 